\title{
TAHSP:
}

The Internet Joưnal of Allied Health Sciences and Practice

A Peer Reviewed Publication of the College of Health Care Sciences at Nova Southeastern University

Dedicated to allied health professional practice and education

http://ijahsp.nova.edu Vol. 11 No. 3 ISSN 1540-580X

\section{The Impact of Body-Scaled Information on Grasping Action in Toddlers with and without Down Syndrome}

\author{
Yu-ping Chen, $\mathrm{ScD}, \mathrm{PT}^{1}$ \\ Allison Mitch, PT, DPT2 \\ Kristen Chafin, PT, DPT2 \\ Rachel Sargent, PT, DPT2
}

1. Assistant Professor, Department of Physical Therapy, Georgia State University, Atlanta, Georgia

2. Doctor of Physical Therapy Student, Department of Physical Therapy, Georgia State University, Atlanta, Georgia

United States

CITATION: Chen Y, Mitch A, Chafin K, Sargent R. The Impact of Body-Scaled Information on Grasping Action in Toddlers with and without Down Syndrome. The Internet Journal of Allied Health Sciences and Practice. July 2013. Volume 11 Number 3.

\begin{abstract}
Background and purpose: Studies of adults and typically developing (TD) children find the number of hands and fingers used to grasp an object is influenced by the relationship between object size and hand size. Children with Down syndrome (DS) between 3 and 11 years have shown no differences in number of hands (1 or 2 hands) used to grasp different sized objects compared to TD children when the object size was body-scaled. The purpose of this study was to examine whether body-scaled information affected the number of hands and fingers used to grasp different sized objects in toddlers with and without DS. Method: Ten toddlers were included, 5 DS $(\bar{x}=16 \mathrm{mo})$ and 5 age-matched TD $(\bar{x}=16.3 \mathrm{mo})$. Subjects were videotaped grasping 14 boxes, $1.4 \mathrm{~cm}$ to $13.5 \mathrm{~cm}$. in size. Number of hands and fingers used for grasping were recorded. Descriptive statistics, t-tests and Spearman correlation coefficient were used to compare the differences between toddlers with DS and TD. Results: Toddlers with DS had significantly smaller hand length than toddlers without DS ( $p=.004$ right hand, $p=.007$ left hand). As box size increased, both groups switched from one-hand to two-hand grasping. There was a significant difference between groups where this transition occurred $(p=.02)$. However, when the body-scaled ratio between object size and hand size was considered, the differences between groups disappeared $(t(8)=1.479 ; p=.18)$. Moreover, the number of fingers used to grasp boxes also increased with increasing box size across both groups $(\rho=0.94)$. Conclusion: Toddlers with DS show similar bodyscaled grasping pattern as their age-matched peers with TD indicating that the differences in grasping patterns between toddlers with and without DS may be attributed to differences in body size, besides the motor abilities.
\end{abstract}

\section{INTRODUCTION}

Human actions emerge from the interaction of the task, the individual, and the environment. ${ }^{1}$ Grasping, or the union of hand(s) and object, is no different and involves those three elements. ${ }^{2}$ There are many ways to grasp an object as a result of the large number of degrees of freedom in the hand and yet still realize the goal of grasping. However, given a particular set of tasks, environment constraints, and object properties, each individual tends to use a preferred grip configuration.

In general, one would expect that with an increase in object size, the number of fingers used to grasp the object would increase, and the chance to use two hands instead of one hand to grasp the object also increases. ${ }^{2}$ Hand size itself may affect preferred grip configurations as a constraint on the individual arising from the individual-task interaction. In other words, an individual with smaller-sized hand may use more fingers or his/her other hand to grasp an object when compared to an individual with larger- 
sized hand. This phenomenon, to adjust their movement performance based on the constraints of their body and the task/environment, is so-called "body scaling."2-5

The relationship between body scaling and grasping pattern (or grip configuration in the article by Newell et al) has been discussed in the populations of typically-developing infants, children, and healthy young adults, demonstrating the influence and predictability of body scaling on grasping patterns. ${ }^{2-4}$ Specifically, one study examining grasping patterns of infants $(n=31$, age 5 months 14 days - 8 months 14 days) and adults ( $n=31$, age 18 to 44 years) using plastic cups of various sizes found that the number of hands and fingers used for grasping in both infants and adults was a function of hand and cup size. ${ }^{2}$ That is, when the ratio of hand size and cup size remained the same, the same grasping pattern was used in both infants and adults. This indicates that body-scaled information can quantitatively describe the relationship between the individual and task constraints.

About 13 of every 10,000 babies born in the US each year have Down Syndrome (DS). ${ }^{6}$ It is widely accepted that individuals with DS not only have intellectual disabilities, but also experience difficulty in performing motor skills. Infants with DS often have a delayed onset of reaching for an interval about 7 weeks. ${ }^{7-9}$ Infants with DS also showed different reaching patterns when comparing with typically developing infants. ${ }^{7-9}$ De Campos and colleagues found that infants with DS had delayed reaching onset with only $43 \%$ of 4-month-old infants with DS in their study starting to reach whereas most of the typically developing infants could reach at 4 months of age. ${ }^{9} \mathrm{~A}$ similar delay was observed in their grasping ability: Hogg and Moss reported children with DS up to 4 years of age displayed fewer mature grasping patterns (i.e. precision grips) than normal children matched with age. ${ }^{10}$ Similar findings were reported by Thombs and Sudgen when testing a variety of grasping tasks in children with DS aged between 6 and 16 years old. Children with DS generally have delayed reaching and grasping skills as compared with their age-matched typical-developing children. ${ }^{11}$

The relationship between object size and grasping pattern in children with DS has been analyzed at older ages.12 Twenty children with DS aged between 4 and 11 years old and age-matched children without DS were presented with boxes of various sizes. It was shown that hand size differed between children with and without DS. This difference in hand size could then be expected to impact grasping patterns; however, once the object size was scaled with their hand size (i.e. body scaling), there was no difference on grasping patterns between children with and without DS. However, this study investigated a group of children at an older age. The examination of hand sizes and grasping patterns has not been found in younger children (i.e. toddlers) with DS. Therefore, the purpose of this pilot study was to examine whether body-scaled information affected the number of hands and fingers used to grasp different sized objects in toddlers with and without DS. If toddlers with DS did not show the similar body scaling as typically-developing toddlers, we could then conclude that this body-scaling required more experience as children at older age practiced grasping for many years. If toddlers with DS showed the similar body scaling like their typically-developing peers, it could be concluded that body scaling emerged regardless grasping experience and age.

\section{METHODS}

Participants

Recruitment for the participants was done between December 2009 and December 2010 by using flyers and emails to local play groups, local Down Syndrome Association, local pediatric physical therapists, and physicians. The inclusion criteria for toddlers with DS were 1) aged between 12 months and 24 months; 2) able to grasp an object for at least 5 seconds; 3) able to see the object presented in front of him/her, and 4) not having other developmental disorders. Ten toddlers were recruited: Five participants were toddlers with DS and the other 5 were typically developing (TD) toddlers who were age matched to toddlers with DS. The mean age for the DS infants was 16.0 months and for the TD infants was 16.3 months. All parents of participants signed the informed consent forms prior to participating this study which was approved by the University IRB.

\section{Apparatus and Procedures}

Testing was arranged with the participant's parents in order to have the most attentive testing time. Nine toddlers were recorded in the laboratory within the university campus while one was recorded at the toddler's home.

A set of 14 stacking boxes of sequential size was used as the stimuli with 10 boxes purchased from a local department store (Circo toys by Target) and 4 boxes constructed out of construction paper and packing tape. All the boxes were filled with beans to make rattle-like sound to attract the infants to grasp. The boxes ranged in size from $1.5 \mathrm{~cm}$ to $13.5 \mathrm{~cm}$, with approximately 1 $\mathrm{cm}$ increase per box. The infant was either seated in an adjustable high chair (Tripp Trapp chair) or in the parent's lap and two video cameras were placed on both sides of the participant during testing. The researcher sat directly in front of the child and the boxes were placed on a table behind the researcher (Fig. 1). 
Figure 1. Experimental Set-up: Camera, Examiner, and Participant Layout

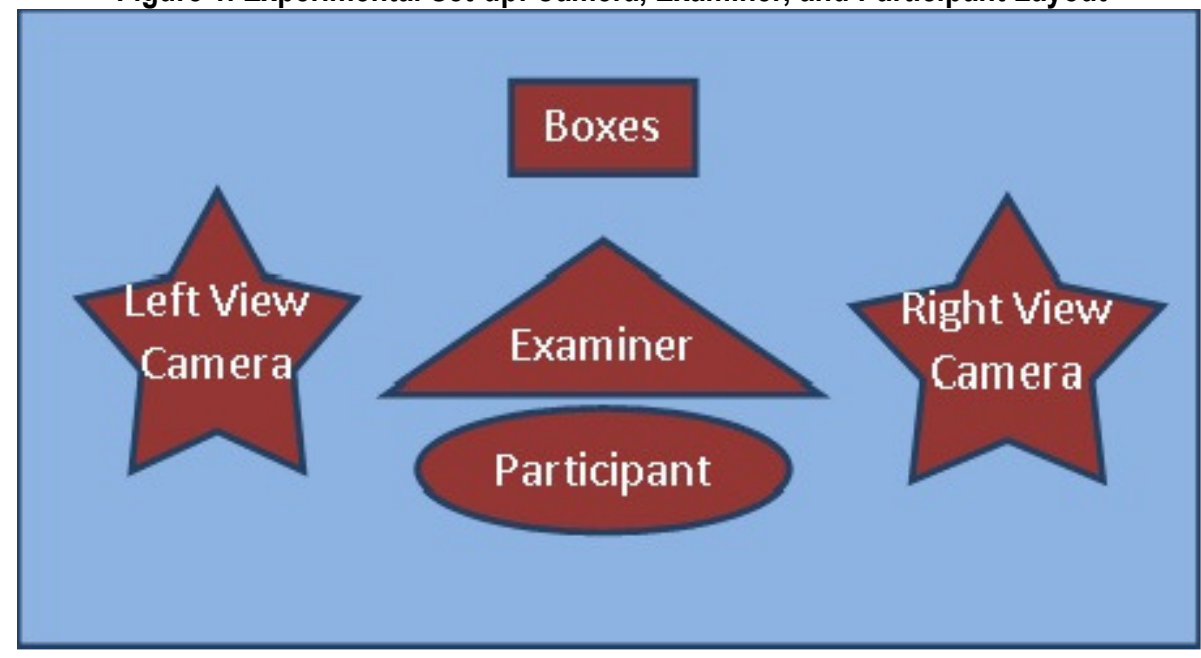

A 5- to 10-minute warm-up/acclimation time was allowed for the toddler if deemed necessary and consisted of playing with a stacking rings set. The boxes were presented to the toddler one at a time, from small to large. This testing sequence was determined to avoid frustration of the toddler as shown by our pilot data. The boxes were placed on a clipboard for presentation to the participant or were placed in the hand of the researcher if the clipboard created a distraction. The examiner always turned her back from the toddler when placing the box on the clipboard or on her hand to avoid the toddler seeing number of fingers used to grasp the box. After picking up the box, the toddler typically either dropped the box on the floor or examined the box. If the toddler showed no interest in the presenting box for 5 seconds, the examiner would shake the box to draw his/her attention. If the toddler still showed no interest in picking up the box after 3 attempts, the examiner would change to the next-sized box and came back to this sized-box at the end of testing. At the end of the testing session, the toddler's hand length (defined as the distance from the distal wrist crease to the tip of the distal phalanx of the third digit) and hand width (defined as the width across the 4 metatarsal heads) were measured using the regular measurement tape.

\section{Data Reduction and Analysis}

All the coding was done by watching the videos using Windows Movie Maker in slow motion by 3 coders. Number of finger used, number of hands used, which fingers used to pick up were coded. All coders received several training sessions to establish their reliability before coding started. During training, all coders received a coding guideline and watched the same video in slow motion together. The coders discussed and determined the number of fingers or hands for each box. If there was any disagreement, the coders would discuss until the consensus reached. At the end of the training session, the reliability for coding hands used to grasp the testing object (1 or 2 hands) was $100 \%$ agreement and number of fingers used was $85 \%$ agreement. All videos were coded by two different coders independently and then their results were compared and discussed. If there was any discrepancy between the two coders, all three coders would meet and obtained the consensus of the coding. Number of fingers and number of hands ( 1 or 2 hands) used were determined at the time each box was picked up by the toddler.

The transition point between one-hand to two-hand grasping followed the method used in the study by Savelsbergh et al. ${ }^{12}$ First, the size of the largest cube $(L)$ that was still grasped with one-hand grasping was determined. Second, the size of the smallest cube (S) that was taken with two hands was determined. Third, the transition point was calculated by the sum of these two cube sizes divided by two $([L+S] / 2)$. The transition point of each toddler was determined and then compared between DS and TD groups.

\section{Statistical Analysis}

Descriptive statistical analyses were performed to describe hand size and number of fingers used for each box. Independent ttests with alpha value at .05 (SPSS version 17) were utilized to examine differences between groups in hand size, the grasp transition point from one-hand to two-hand, and grasping patterns with actual box sizes and with body-scaled box sizes. The association between number of fingers and box sizes was also examined using the Spearmen correlation coefficient.

\section{RESULTS}

The average percentage of completion of the 14-sized boxes between toddlers with DS and TD was $77 \%$ and $96 \%$, respectively. Toddlers with DS got distracted easily and had a lower completion rate. 
Participants' demographics are listed in Table 1. Toddlers with DS in our study had significantly smaller hand length than toddlers without DS (t (8)=3.913, $p=.004$ for right hand, $t(8)=3.614, p=.007$ for left hand).

Table 1. Demographic Characteristics and Anthropometric Measures of the Participants

\begin{tabular}{|l|l|l|l|l|l|}
\hline id & Group & $\begin{array}{l}\text { Age (in months } \\
\text { and days) }\end{array}$ & Gender & Hand length (cm) & Hand width (cm) \\
\hline 1 & DS & $18 \mathrm{M} 6 \mathrm{D}$ & $\mathrm{F}$ & 7.25 & 4.85 \\
\hline 2 & DS & $14 \mathrm{M} 18 \mathrm{D}$ & $\mathrm{F}$ & 8.00 & 5.75 \\
\hline 3 & DS & $11 \mathrm{M} 9 \mathrm{D}$ & $\mathrm{M}$ & 7.55 & 4.50 \\
\hline 4 & DS & $13 \mathrm{M} 9 \mathrm{D}$ & $\mathrm{F}$ & 7.25 & 4.15 \\
\hline 5 & DS & $23 \mathrm{M} 18 \mathrm{D}$ & $\mathrm{F}$ & 8.00 & 4.70 \\
\hline 6 & TD & $19 \mathrm{M} 9 \mathrm{D}$ & $\mathrm{F}$ & 9.70 & 5.10 \\
\hline 7 & TD & $13 \mathrm{M} 0 \mathrm{D}$ & $\mathrm{M}$ & 8.65 & 4.50 \\
\hline 8 & TD & $10 \mathrm{M} 26 \mathrm{D}$ & $\mathrm{F}$ & 8.25 & 4.35 \\
\hline 9 & TD & $14 \mathrm{M} 20 \mathrm{D}$ & $\mathrm{F}$ & 9.8 & 4.75 \\
\hline 10 & TD & 23M25D & M & 10.3 & 5.35 \\
\hline
\end{tabular}

DS: Down Syndrome; TD: Typical Development

\section{Hands}

As box size increased, both groups switched from one-hand to two-hand grasping (Fig. 2). There was a statistically significant difference between where this transition occurred between groups $(t(8)=2.832, p=.02)$. In the group of toddlers with DS, the majority of participants $(75 \%)$ transitioned to using two hands to grasp the $7^{\text {th }}$ box $(5.9 \mathrm{~cm})$, and the majority of typically developing group (80\%) did not switch to use of two hands until the $9^{\text {th }}$ box $(8.1 \mathrm{~cm})$.

When the body-scaled ratio between object size and hand size was considered, the between group difference disappeared $(t(8)=1.479, p=.18)$ (Fig. 3). This data shows the majority of both groups (approximately $80 \%$ frequency) switched to using two hands at a comparable box size to hand length ratio (toddlers with $\mathrm{DS}=0.77$; toddlers with $T D=0.87$ ).

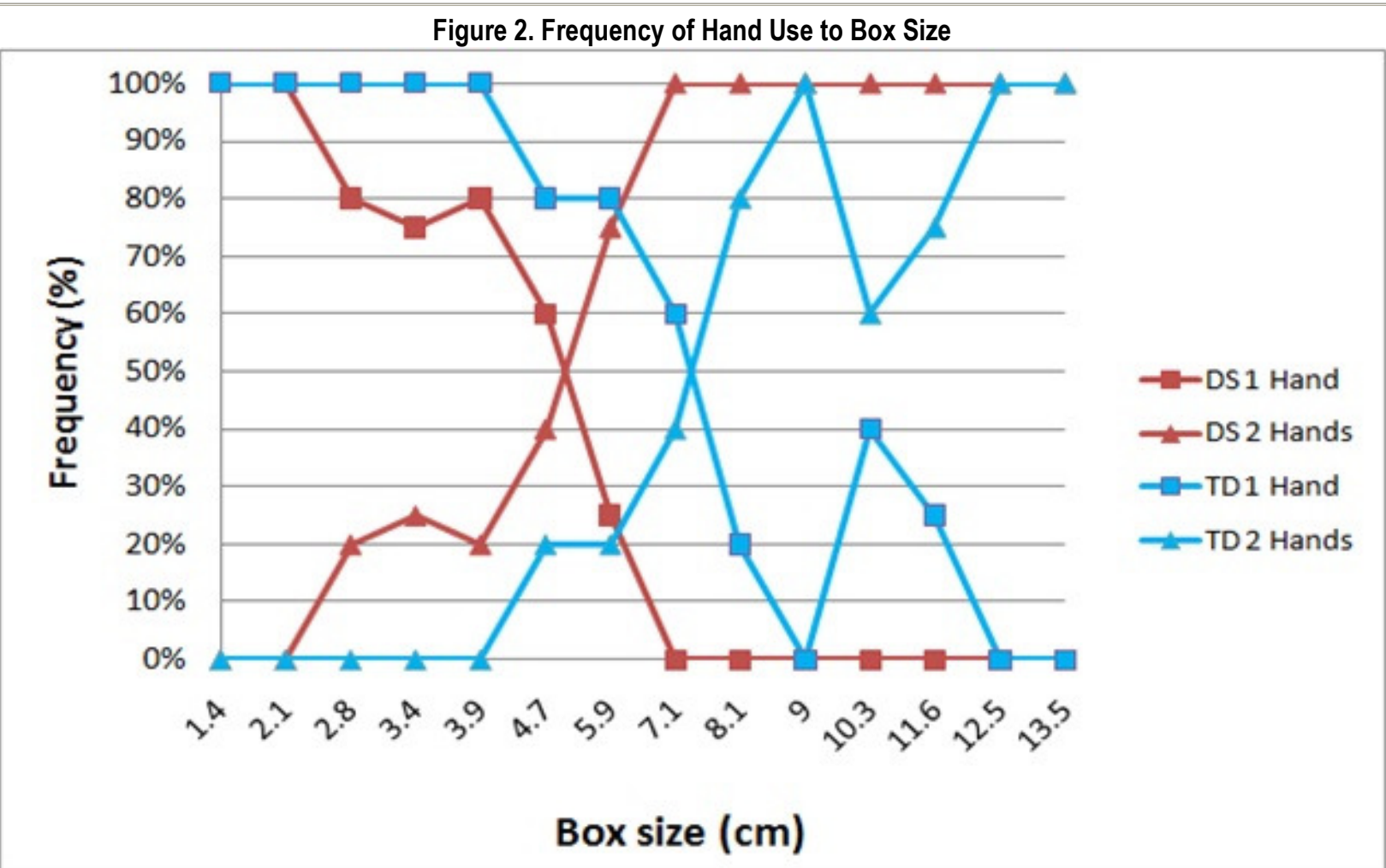

${ }^{*}$ Frequency refers to the percentage of that sample that used either 1 or 2 hands 
Figure 3. Frequency of Hand Use to Body-Scaled Ratio

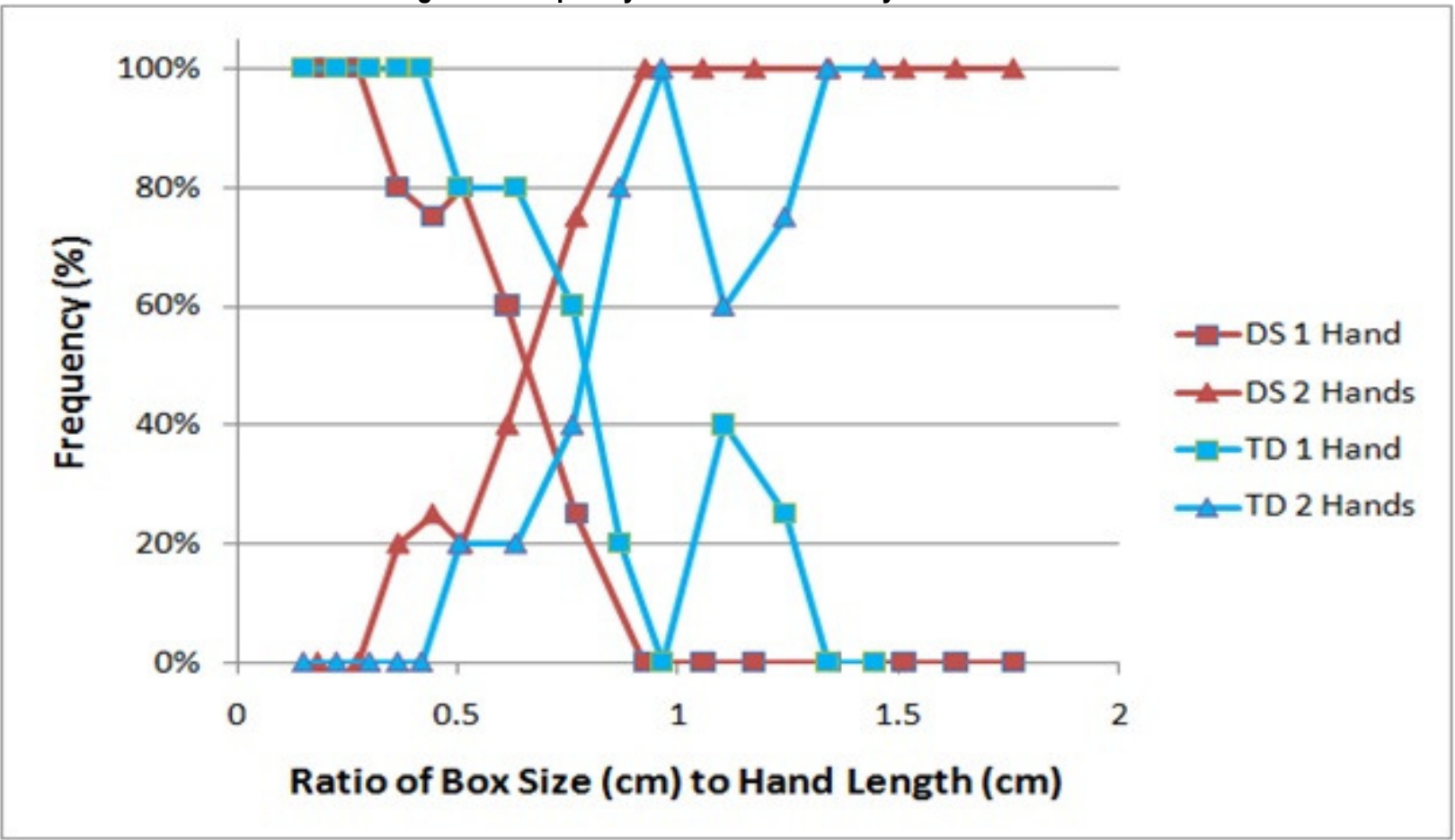

\section{Fingers}

In addition to the number of hands used, the number of fingers used was examined, and the number of fingers used was found to increase with increasing box size (Fig. 4). Considering all the data across both groups of toddlers, a strong association was found between number of fingers used and object size (Spearman $p=0.94$ ). These results depict the increasing finger number with object size for both groups using the body scaled data.

Figure 4. Number of Digits Used to Box Size

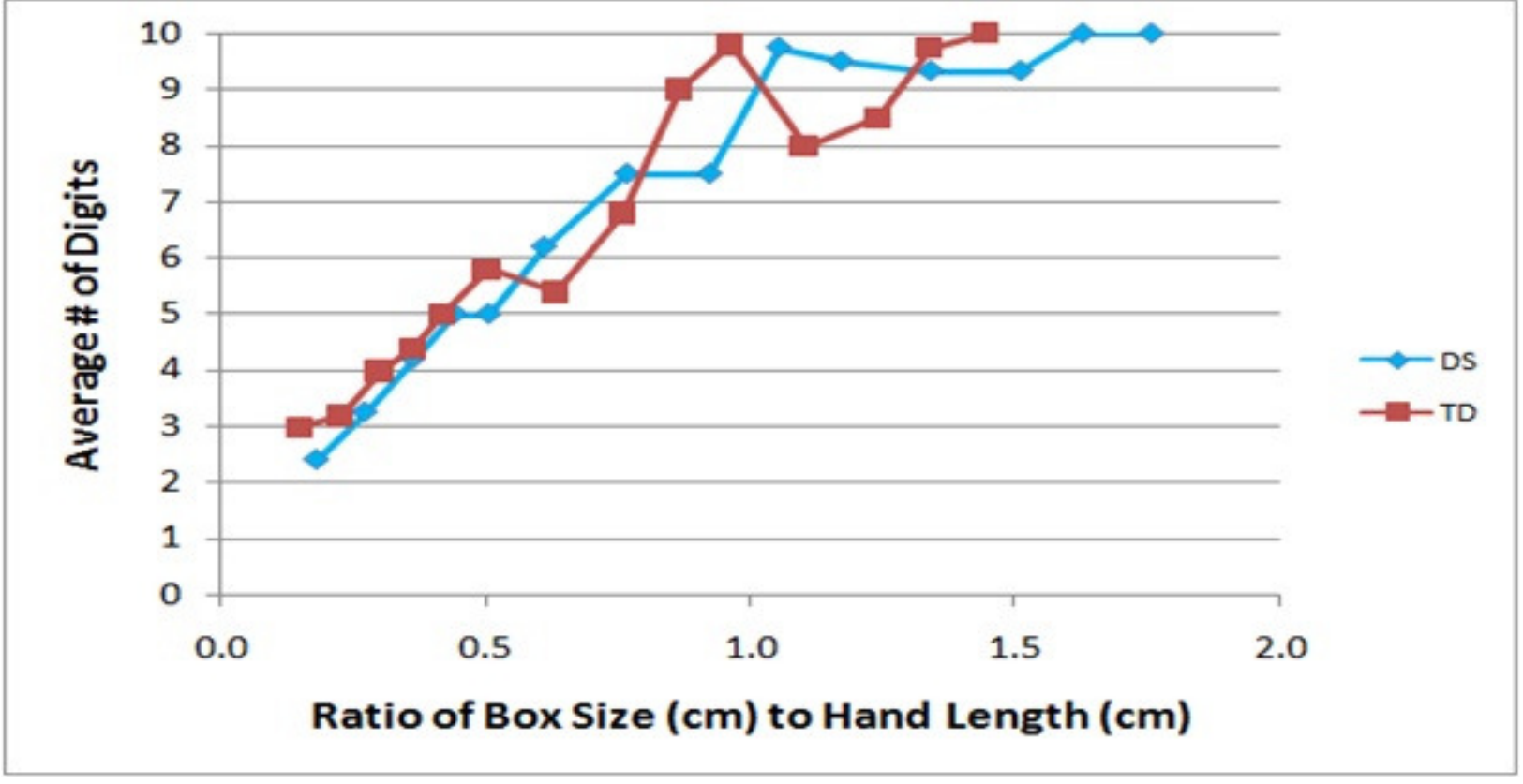




\section{DISCUSSION}

The findings of this pilot study demonstrate the strong role body scale plays in determining grip configuration (number of hands use and number of fingers use) in toddlers to changes in object size, regardless their genetic abnormalities and intellectual disabilities (i.e. Down Syndrome). Toddlers with DS show similar body-scaled grasping patterns as their age-matched peers with TD. This finding is consistent with other studies that found that typically developing children and adults have similar grasping patterns when body-scale is considered. ${ }^{2}$ This finding is also consistent Savelsberghet al who found that older children and adolescents with DS have similar body-scaled grasping patterns as their same aged TD peers. ${ }^{12}$ That is, toddlers with DS do not require lots of practice experience in reaching and grasping to acquire body-scaling. Our finding just confirms the dimensionless ratio (or body-scaled) in the perception action framework. 13-17 Grasping is an emergent action that depends on the constraints of the individual (i.e. hand size) and the individual's fit within environmental constraints (i.e. object size).

The transition point from one-hand to two-hand grasping in our study was 0.77 and 0.87 for the DS and TD group respectively, which was lower than the ratios reported by Savelsbergh et al (0.98) but higher than the study reported by Newell et al $(0.60) .2,12$ One possible explanation, similar to Savelsbergh et al (2001), was the difference in the "compressibility" of the box material. ${ }^{12}$ Our box set was more rigid than the regular cardboard paper used in by Savelsbergh et al but less rigid than the plastic material used in Newell's study. Another possible explanation was the way to measure hand size. Our method followed the method used by Newell et al to measure between the distal wrist crease to the tip of the distal phalanx of the third digit, whereas Savelsbergh et al used the distance between thumb and index finger span, which result in the smaller numerical ratio in our study as compared with Savelsbergh et al.2,12

The clinical implication of our findings is to be cautious in determining the fine motor delay in infants/toddlers with DS, as the evaluation toolkits in most of the standardized assessment tests use the one-sized objects (e.g. 1-inch cube). Infants/toddlers with DS have statistically significantly smaller hand sizes than their age-matched peers as indicated by our pilot work and that of others. The grasping patterns shown in infants/toddlers with DS might still use the "immature" grasping, such as power grasping rather than pincer grasping, which may not purely be due to their cognition deficits or impaired motor planning abilities, but also hand size differences. That is, if providing a smaller-sized of object when testing grasping, a different skill level may be revealed when testing infants/toddlers with DS. Valid evidence has been provided by Newell et al. ${ }^{2}$ They examined grasping patterns of infants ( $n=31$, age 5 months 14 days - 8 months 14 days) and adults $(n=31$, age 18 to 44 years) using plastic cups of various sizes and found that the number of hands and fingers used for grasping in both infants and adults was a function of hand and cup size. That is, young infants used 2-3 fingers to grasp smaller cups (i.e. pincer grasping), the mature grasping pattern which is not seen until 12 months of age or later. Once the object size scaled down, a "mature" pincer grasping can be observed.

Interestingly, a lack of variability in grasping patterns of the DS group as compared to the TD group was observed. Once the DS group switched to a two-handed grasping pattern, they all continued to use this strategy for all subsequent boxes. The TD group, however, showed variability in grasping pattern as they were able to switch between a one-handed and two-handed pattern. Figure 2 shows the consistency of two handed used in the DS group. All participants with DS switched to two hands (that is a $100 \%$ frequency) by the $8^{\text {th }}$ box $(7.1 \mathrm{~cm})$ and maintained a two-handed pattern. The TD group first reached a $100 \%$ two-hand use frequency at the $10^{\text {th }}$ box $(9 \mathrm{~cm})$, but showed variability over the next two boxes at inconsistent frequency secondary to 2 individuals using 1 hand. Two-handed use returned to $100 \%$ frequency for box the $13^{\text {th }}$ and $14^{\text {th }}$ boxes. This observation is consistent with publications investigating how variability in motor patterns is necessary to allow for flexibility, exploration, learning, and adaptation; lack of variability, or inability to select a strategy out of the movement repertoire, is indicative of atypical motor development. ${ }^{18-20}$

This research has a variety of implications for physical therapists. When evaluating a child with DS, it should be considered that a delay in prehension may be due to not only cognition or motor planning abilities, but also their smaller hand size. When working on grasp/prehension, it may be necessary to use smaller objects/toys to allow the child to more accurately demonstrate ability level and represent their grasping pattern, and activities which encourage problem solving and variability in grasping should be used. Additionally, when observing a child who demonstrates a lack of variability in age appropriate motor planning, you may consider they have some atypical motor development.

This study was hindered in statistical power by its limited sample size secondary to difficulty recruiting subjects within our timeframe limitations. Future research is needed on the role of body scaling in individuals with DS and should include a larger sample size. Also, due to the short attention span in the toddlers with DS in our pilot study, each object was tested only once, which might limit the study's generalizability, even though each child was tested at his/her most alert period of the time. Future studies may consider testing toddlers with DS in repeated sessions with relatively short testing sessions to get more data collected.

(c) The Internet Journal of Allied Health Sciences and Practice, 2013 


\section{CONCLUSION}

Toddlers with DS show similar body-scaled grasping patterns as their age-matched TD peers. When evaluating a child with DS, it should be considered that a delay in prehension may be due to not only cognition or motor planning abilities, but also hand size.

\section{ACKNOWLEDGEMENTS}

This study was partially funded by the College of Health and Human Sciences Intramural Research Funding, Georgia State University, to the first author (Y.Chen). The authors want to acknowledge Oh Baby Fitness!, Play 2 Grow, Down Syndrome Association of Atlanta, and Dr. J. Visootsak at Emory University for their assistance in recruitment of participants. They also want to acknowledge the toddlers and their family for their participation.

\section{REFERENCES}

1. Newell KM. Constraints on the development of coordination. In MG Wade \& HTA Whiting (eds.), Motor development in children: Aspects of coordination and control. Boston: Martinus Nijhoff; 1986:341-60.

2. Newell KM, McDonald PV, Baillargeon R. Body scale and infant grip configurations. Dev Psychobiol. 1993;26(4):195-205. [PMID 8354425]

3. Cesari P, Newell KM. Body scaling of grip configurations in children aged 6-12 years. Dev Psychobiol. 2000;36(4):301-10. [PMID 10797251]

4. Ceasri P, Newell KM. Body-scaled transitions in human grip configurations. J Exp Psychol Hum Percept Perform. 2000;26(5):1657-68. [PMID 11039491]

5. Cesari P, Newell KM. The scaling of human grip configurations. J Exp Psychol Hum Percept Perform.. 1999;25(4):927-35. [PMID 10464939]

6. Centers for Disease Control and Prevention (CDC). Down Syndrome. Accessed 9/17/2012.

7. Cunningham CC. Aspects of early development in Down syndrome infants. Unpublished PhD Dissertation, University of Manchester; 1979.

8. Cadoret G, Beuter A. Early development of reaching in Down syndrome infants. Early Hum Dev. 1994;36(3):157-73. [PMID 8062782]

9. de Campos AC, Rocha NA, Savelsbergh GJ. Development of reaching and grasping skills in infants with Down syndrome. Res Dev Disabil. 2010;31(1):70-80. doi: 10.1016/j.ridd.2009.07.015. [PMID 19713074]

10. Hogg J, Moss SC. Prehensile development in Down's syndrome and non-handicapped preschool children. British J Dev Psy. 1983;1:189-204.

11. Thombs B, Sugden D. Manual skills in Down syndrome children ages 6 to 16 years. Adapt Phys Act Q. 8(3):242-54.

12. Savelsbergh GJP, van der Kamp J, Davis WE. Perception-action coupling in grasping of children with Down syndrome. Adapt Phys Act Q. 2001;18(4):451-7.

13. Gibson J. The Ecological Approach to Visual Perception. Boston, Mass: Houghton Mifflin; 1979.

14. Gibson J. The Senses Considered as Perceptual Systems. Boston, Mass: Houghton-Mifflin; 1966.

15. Gibson EJ, Pick AD. An Ecological Approach to Perceptual Development. An Ecological Approach to Perceptual Learning and Development. NY: Oxford University Press; 2000:14-25.

16. Gibson EJ. An ecological psychologist's prolegomena for perceptual development: A functional approach. In: ZukowGoldring CD-RP, ed. Evolving Explanations of Development: Ecological Approaches to Organism-Environment Systems. Washington, DC: APA; 1997.

17. Fetters L, Ellis T. A perception-action framework for physical therapy for persons with neurologic dysfunction: Use of therapeutic affordances and unitless ratio. J Neurologic Phys Ther. 2006;30(3):142-7. [PMID 17029657]

18. Hadders-Algra M. Variation and variability: keywords in human motor development. Phys Ther. 2010;90:1823-37. [PMID 20966209]

19. Vereijken B. The complexity of childhood development: Variability in perspective. Phys Ther. 2010;90(12):1850-9. doi: 10.2522/ptj.20100019. [PMID 20966207]

20. Fetters L. Perspective on variability in the development of human action. Phys Ther. 2010 Dec;90(12):1860-7. doi: 10.2522/ptj.2010090. [PMID 20966211]

\section{KEY TERMS}

Down Syndrome, Toddlers, Grasp, Object Size, Body Scale 